\title{
THE EFFECT OF CHROMOSOME 5B ON SYNAPSIS AND CHIASMA FORMATION IN WHEAT, TRITICUM AESTIVUM Cv. CHINESE SPRING
}

\author{
by \\ PREBEN BACH HOLM ${ }^{1)}$ and XINGZHI WANG ${ }^{1,2)}$ \\ 1"Department of Physiology, Carlsberg Laboratory, \\ Gamle Carlsberg Vej 10, DK-2500 Copenhagen Valby \\ ${ }^{2}$ Institute of Genetics, Academia Sinica, Beijing, China
}

Keywords: Ph, crossing over, allopolyploidy, homoeologue

\begin{abstract}
The present paper summarizes and discusses the results obtained from a series of ultrastructural investigations of the effect of chromosome 5B on chromosome pairing and synaptonemal complex formation in wheat, $\mathrm{T}$. aestivum cv. Chinese Spring. The material comprises hexaploid wheat nullisomic for chromosome 5B, monosomic for $5 \mathrm{~B}$, euploid wheat, wheat where chromosomes $5 \mathrm{~B}$ have been replaced by one, two or three copies of an isochromosome for the long arm of chromosome $5 \mathrm{~B}$; trihaploid wheat with and without $5 \mathrm{~B}$ and hybrids between Chinese Spring wheat and diploid rye with chromosome $5 \mathrm{~B}$, without $5 \mathrm{~B}$ or carrying an isochromosome for the long arm of 5B. It is shown that nullisomy for chromosome $5 \mathrm{~B}$ in hexaploid wheat results in a two- to threefold increase in the number of lateral components in multiple associations already from the beginning of zygotene and in trihaploid wheat and wheat-rye hybrids in a two- to threefold increase in the number of pairing partner exchanges per lateral component involved in synapsis. Only nullisomy for chromosome 5B and triisosomy for the long arm of chromosome $5 \mathrm{~B}$ permit crossing over between the homoeologous chromosomes of wheat. It is inferred that the product of the $\mathrm{Ph}$ locus on chromosome 5B affects both synapsis and crossing over. As these timewise separated processes are assumed both to be initiated by heteroduplex formation, this might provide the common link in the action of the $\mathrm{Ph}$ gene. The results obtained with the genotypes containing different dosage of the $\mathrm{Ph}$ gene disprove both the hypothesis that $\mathrm{Ph}$ influences a premeiotic chromosome rearrangement $(13,14,15)$ and the hypothesis $(18,82)$ that $\mathrm{Ph}$ controls the timing of crossing over with respect to the process of correction of multiple pairing associations.
\end{abstract}

\section{INTRODUCTION}

In $1917 \varnothing$. WINGE (83) proposed that hybridization between closely related species followed by chromosome doubling was a common event in the evolution of higher plants. Thereby, as discussed by WINGE, the frequent occurrence of arithmetic series of chromosome numbers found in many plant genera could be explained and each chromosome would as a result of chromosome doubling be provided with a homologous partner during meiosis. Hybridization followed by chromosome doubling, later referred to as allopolyploidy (37) or am- phidiploidy (47) is now generally accepted as a major evolutionary mechanism and it is estimated that up to $50 \%$ of all higher plants, including some of the most important crop plants (e.g. wheat, cotton, tobacco and oats) are allopolyploids $(5,60,64,65,72)$. Recent molecular and genetic studies have also suggested that at least hybridization may be an important factor in the evolution of lower eukaryotes such as yeasts, as the lager brewing yeast, Saccharomyces carlsbergensis, appears to have a hybrid origin $(48,50)$.

The establishment of a new allopolyploid 
species is dependent on a number of parameters, a major one being diploid behaviour at meiosis. The stability of the species as well as its fertility depends on crossing over being confined to homologous chromosomes, as crossing over between the partly homologous (homoeologous) chromosomes of the parental genomes results in the breakdown of the individual genomes as well as partial sterility due to the irregular segregation of the chromosomes in multivalents at the first meiotic division.

As allopolyploids often are considered superior agronomically to their diploid ancestors, due to the formation of larger plants, flowers, fruits, seeds, greater ecological adaptability and greater evolutionary potential because of their permanent hybrid character (3), numerous attempts to create new allopolyploids have been made. However, with a few exceptions such attempts have failed, primarily because of the occurrence of crossing over between the homoeologous chromosomes. This implies that allopolyploids in the course of their evolution have acquired a meiosis which insures a disomic inheritance. As allopolyploidy is common throughout the evolutionary spectrum of higher plants, a mechanism for exclusive chiasma formation between homologous chromosomes must have evolved independently in a number of species $(5,60,65)$.

Cytogenetic studies of the common bread wheat, Triticum aestivum, have provided some insight into this. T. aestivum is generally considered a type example of an allopolyploid and extensive investigations have shown that it is an allohexaploid $(2 n=6 x=42)$ which has arisen by two successive hybridization events and chromosome doublings, involving three different species (T. monococcum, $2 x=14$, A genome:, unknown species, $2 x=14, B$. genome; $T$. tauschii, $2 x=14$, D genome). Breeding experiments and genetic and cytological studies have shown that each pair of chromosomes is homoeologous to a chromosome pair in each of the two other genomes. Irrespective of this partial homology between the three genomes, crossing over and chiasma formation occur only between homologues, the outcome being an exclusive presence of bivalents at metaphase $I$, a regular segregation and a disomic inheritance $(60,64$, $65)$.
Cytogenetic studies have disclosed the presence of a major controlling gene ( $\mathrm{Ph}$, pairing homoeologous) in the middle of the long arm of chromosome 5B $(10,29,66)$. This gene is generally considered to be a single locus, as there is no segregation for intermediate meiotic characteristics in the progeny, although as discussed by SEARS (65) the presence of two or more loci cannot be excluded. In the absence of chromosome $5 \mathrm{~B}$ or its long arm or in the ph $1 \mathrm{~b}$ mutant line (66) the disomic inheritance breaks down due to crossing over and chiasma formation between the homoeologues in hexaploid wheat, in the trihaploid or in hybrids between wheat and related species $(38,49,56,57,59,66)$. By crossing wheat nullisomic for chromosome $5 \mathrm{~B}$, or carrying the ph $1 \mathrm{~b}$ mutation, with other wheat species or rye, various genes conferring disease resistance have been transferred into $T$. aestivum by means of meiotic crossing over between the wheat chromosomes and their homoeologues from the other species participating in the cross $(61,68)$.

Additional genes of minor importance for regulating the diploid like meiosis of $T$. aestivum have been identified on the short arms of homoeologue group 3. The absence of these chromosome arms leads to chiasma formation between the homoeologues in hybrids between wheat and related species, but has no effect on meiosis in hexaploid wheat $(28,43,44,45)$. The effect of the short arm of chromosome $3 \mathrm{D}$ is attributed to a locus $\mathrm{Ph} 2$ (67) formerly believed to reside in the long arm of chromosome $5 \mathrm{~B}(77$, 78). Finally, $\mathrm{Ph}$ like genes also appear to be present in accessory $\mathrm{B}$ chromosomes of Aegilops mutica and A. speltoides, since the presence of these chromosomes in hybrids between $T$. aestivum and these Aegilops species counteract the effect of nullisomy for chromosome 5B (6).

Ultrastructural studies of euploid wheat using serial sectioning and three dimensional reconstruction $(18,30)$ or spreading and silver staining of meiotic nuclei $(20)$ revealed that chromosome pairing and synaptonemal complex formation at zygotene primarily occurred between homologues. However, in most nuclei (8 of 11 mid zygotene and 4 of 7 late zygotene nuclei (20)) one or more multiple associations had formed due to pairing partner exchanges. Nearly 
all multiple associations were corrected before pachytene, but a few persisted through this stage where crossing over occurs. These observations led to the general proposal that the disomic inheritance in allohexaploid wheat can be attributed to the following (20):

1) A high stringency of synapsis during zygotene, whereby synaptonemal complex formation primarily occurs between homologues.

2) A correction of chromosome pairing at the end of zygotene, whereby multiple associations are transformed into pairs of homologues, bivalents, before crossing over occurs.

3) Suppression of crossing over between paired segments of homoeologous chromosomes retained during the crossing over period.

The ultrastructural investigations now comprises studies of chromosome pairing in trihaploid wheat with and without chromosome 5B (79), wheat-rye hybrids nullisomic for 5B, euploid hybrids and hybrids where chomosome $5 \mathrm{~B}$ has been replaced by an isochromosome for the long arm of chromosome 5B (monoisosomic $5 B L)(80)$, hexaploid wheat nullisomic for $5 \mathrm{~B}$ (22), monosomic for $5 \mathrm{~B}(21)$, euploid wheat ( 18 , $20,31)$, and wheat monoisosomic and diisosomic for 5BL (23) and triisosomic for 5BL (84). These studies were all performed on the cultivar Chinese Spring. In addition, trihaploids derived from the Chinese winter wheat varity Kedong have been analyzed (79) and exploratory studies have been performed on two hexaploid Danish winter wheat varieties Vuka and Kraka (23). The results obtained for the different genotypes are given in the individual papers, but it is the intention of the present paper to summarize the most important findings from these studies and with their aid discuss the function of the $\mathrm{Ph}$ locus on the long arm of chromosome 5B.

\section{MATERIALS AND METHODS}

The materials, the growth conditions and the procedures for light and electron microscopy are described in the individual reports in this series $(18,20,21,22,23,30,79,80,84)$.

\section{RESULTS}

\subsection{General aspects}

Initiation and progression of chromosome pairing and synaptonemal complex formation during zygotene were monitored to assess if different dosages of chromosome $5 \mathrm{~B}$ or its long arm have qualitative and/or quantitative effects on synapsis. The specificity of chromosome pairing and synaptonemal complex formation is evaluated through counting the number of lateral components which by pairing partner exchange are involved in multiple associations or by directly counting the number of pairing partner exchanges per lateral component (haploid wheat and wheat-rye hybrids). The degree of synapsis achieved is measured in pachytene nuclei or nuclei where synaptonemal complex degradation has just been initiated (nuclei at the pachytene to diplotene transition or early diplotene nuclei).

\subsection{Hexaploid wheat}

From the summary of the data in Table I the following conclusions can be drawn as to the effect of chromosome 5B:

1) Chromosome $5 B$ affects the extent of chromosome pairing and synaptonemal complex formation. In its absence synaptonemal complex formation stops when $35-40 \%$ of the complement has synapsed (pachytene: mean of $42 \%$, pachytene to diplotene transition: mean of $37 \%$, and early diplotene: mean of $33 \%$ ). Monosomy for chromosome $5 \mathrm{~B}$ results in synaptonemal complex formation for $87 \%$ of the complement (pachytene: mean of $90 \%$, early diplotene: mean of $84 \%$ ) while in euploid wheat synapsis is slightly more complete $(91 \%$; pachytene: mean of $97 \%$, early diplotene: mean of $85 \%$ ). The replacement of chromosome 5B with one copy of an isochromosome for the long arm of $5 \mathrm{~B}$ results in pairing arrest when $55 \%$ of the complement has synapsed (23), two isochromosomes lead to arrest when $40 \%$ of the complement is joined with a synaptonemal complex while the presence of three copies of the isochromosome supports even less synapsis (25\%). As discussed in section 4.2 the arrest of synapsis in nullisomic $5 \mathrm{~B}$ wheat or the genotypes carrying the isochromosomes results from the lack of the short arm of chromosome 5B.

2) There is an apparent correlation between the dosage of chromosome 5B and the specificity of synapsis, expressed as the number of lateral 
Table I. Degree of pairing, lateral component (LC) length of one complement $(1 / 2, L C$ length) and the number of lateral components or lateral component segments involved in multiple associations at early-mid zygotene, mid-late zygotene, pachytene, and early diplotene in wheat nullisomic for chromosome $5 B$ $(0 \times 5 B)$, monosomic for $5 B(1 \times 5 B)$, euploid wheat $(2 \times 5 B)$, wheat diisosomic $(4 \times 5 B L)$ and triisosomic (6x5BL) for the long arm of chromosome 5B. The number of multiple associations in parentheses are associations of type II. ND, not determined. \pm , standard deviation.

\begin{tabular}{llllll}
\hline $\begin{array}{l}\text { Early-mid zygotene } \\
\text { Geno- } \\
\text { type }\end{array}$ & $\begin{array}{l}\text { Num- \% } \\
\text { ber of pair- } \\
\text { nuclei ing }\end{array}$ & $\begin{array}{l}1 / 2 \text { LC } \\
\text { length } \\
\mu \mathrm{m}\end{array}$ & \multicolumn{2}{l}{$\begin{array}{l}\text { Number of } \\
\text { LC's in } \\
\text { multiple } \\
\text { associations }\end{array}$} \\
\hline $0 \times 5 \mathrm{~B}$ & 3 & 20 & 2691 & 25 & $(6)$ \\
$1 \times 5 \mathrm{~B}$ & 3 & 42 & 2342 & 15 & $(4)$ \\
$2 \times 5 \mathrm{~B}$ & 5 & $39 \pm 7$ & $1984 \pm 342$ & $8 \pm 4$ & $(6 \pm 3)$ \\
$4 \times 5 \mathrm{BL}$ & 7 & $31 \pm 6$ & $1940 \pm 222$ & $6 \pm 5$ & $(2 \pm 4)$ \\
$6 \times 5 \mathrm{BL}$ & 1 & 25 & 1722 & 32 & 0 \\
\hline
\end{tabular}

Mid-late zygotene

\begin{tabular}{lccll}
\hline $0 \times 5 \mathrm{~B}$ & 5 & $43 \pm 6$ & $2292 \pm 425$ & $17 \pm 9(7 \pm 4)$ \\
$1 \times 5 \mathrm{~B}$ & 6 & $79 \pm 8$ & $1887 \pm 254$ & $10 \pm 4(0)$ \\
$2 \times 5 \mathrm{~B}$ & 10 & $62 \pm 7$ & $2040 \pm 164$ & $5 \pm 6(3 \pm 3)$ \\
$2 \times 5 \mathrm{~B} \S$ & 7 & $93 \pm 7$ & $1806 \pm 160$ & $5 \pm 5(0)$ \\
$4 \times 5 \mathrm{BL}$ & ND & ND & ND & ND \\
$6 \times 5 \mathrm{BL}$ & ND & ND & ND & ND \\
\hline
\end{tabular}

Pachytene

\begin{tabular}{llllll}
\hline $0 \times 5 \mathrm{~B}$ & 4 & $42 \pm 13$ & $1672 \pm 142$ & $17 \pm 6$ & $(6 \pm 6)$ \\
$0 \times 5 \mathrm{~B} \S \S$ & 6 & $37 \pm 15$ & $1722 \pm 177$ & $\mathrm{ND}$ & \\
$1 \times 5 \mathrm{~B}$ & 17 & $90 \pm 5$ & $1322 \pm 220$ & $9 \pm 3$ & $(1)$ \\
$2 \times 5 \mathrm{~B}$ & 20 & $97 \pm 7$ & $1474 \pm 220$ & 0.6 & $(0.3)$ \\
$4 \times 5 \mathrm{BL}$ & $\mathrm{ND}$ & $\mathrm{ND}$ & $\mathrm{ND}$ & $\mathrm{ND}$ & \\
$6 \times 5 \mathrm{BL}$ & $\mathrm{ND}$ & $\mathrm{ND}$ & $\mathrm{ND}$ & $\mathrm{ND}$ & \\
\hline
\end{tabular}

Early diplotene

\begin{tabular}{lrllll}
\hline $0 \times 5 \mathrm{~B}$ & 7 & $33 \pm 17$ & $1722 \pm 360$ & ND & \\
$1 \times 5 \mathrm{~B}$ & 4 & $84 \pm 5$ & $1354 \pm 271$ & 10 & $(0)$ \\
$2 \times 5 \mathrm{~B}$ & 12 & $85 \pm 14$ & $1108 \pm 192$ & ND & \\
$4 \times 5 \mathrm{BL}$ & 6 & $40 \pm 12$ & $1702 \pm 185$ & ND & \\
$6 \times 5 \mathrm{BL}^{\$}$ & 3 & 15 & 1408 & ND & \\
\hline
\end{tabular}

$\S$, Data for mid zygotene and for late zygotene. $\$$, Data for the pachytene to diplotene transition. $\$$, Data for early-mid diplotene components involved in multiple associations by pairing partner exchange (Table I). Throughout zygotene there is thus a 3:2:1 ratio between the number of lateral components in multiple associations in hexaploid wheat carrying 0,1 or 2 copies of chromosome $5 \mathrm{~B}$. In plants with two isochromosomes for the long arm of chromosome $5 \mathrm{~B}$, the number of pairing partner exchanges between chromosomes that are not homologous may be even lower than in euploid wheat while there is a drastic increase in the number of pairing partner exchanges in triisosomic 5BL wheat. The correlation between copy number of chromosome $5 \mathrm{~B}$ or its long arm and the frequency of pairing partner exchanges is discussed in section 4.3.

3) There is an effect of chromosome $5 B$ on the type of pairing partner exchange. At early and mid zygotene in euploid wheat most pairing partner exchanges are of type II, i.e. associations consisting of two partially paired bivalents or a bivalent and a univalent, which synapses with each other over a 0.5-1.0 $\mu \mathrm{m}$ distance, whereafter the involved lateral components switch back to their original partner. In nullisomic and monosomic 5B wheat more complex associations form and single pairing partner exchanges with long segments of synaptonemal complex around the region of exchange are much more frequent. Only in nullisomic 5B wheat do the chromosomes exchange partners close to the telomeres while in the other genotypes the exchanges are almost exclusively in interstitial chromosome segments.

4) In euploid wheat almost all multiple associations are resolved into bivalents by the synaptic correction mechanism before pachytene while in nullisomic and monosomic 5B wheat the multiple associations present at mid-late zygotene persist uncorrected through pachytene and into diplotene. These differences in synaptic correction are discussed in section 4.4.

5) Irrespective of the presence of several multiple associations at pachytene in monosomic 5B wheat and a few in euploid wheat and probably also in diisosomic 5BL wheat, crossing over and chiasma formation are exclusively confined to homologous pairs in the three genotypes while crossing over and chiasma formation can occur between homoeologues in the absence of chro- 
Table II. Degree of pairing, number of lateral components (LC) in multiple associations involving more than three LC's, number of pairing partner exchanges per LC engaged in synapsis and number of chiasmata per metaphase $I$ in trihaploid wheat with $(1 \times 5 B)$ and without $(0 \times 5 B)$ chromosome $5 B$.

\begin{tabular}{lccccc}
\hline Genotype & $\begin{array}{c}\text { Number } \\
\text { of } \\
\text { nuclei }\end{array}$ & $\begin{array}{c}\% \\
\text { pairing }\end{array}$ & $\begin{array}{c}\text { Number of LC's } \\
\text { in multiple } \\
\text { associations }\end{array}$ & $\begin{array}{c}\text { Number of pairing } \\
\text { partner exchanges } \\
\text { per LC }\end{array}$ & $\begin{array}{c}\text { Number of } \\
\text { chiasmata }\end{array}$ \\
\hline $0 \times 5 \mathrm{~B}$ & 2 & 14.4 & 29 & 0.57 & 6.73 \\
$1 \times 5 \mathrm{~B}$ & 12 & $10-20$ & 12 & 0.37 & 0.16 \\
\hline
\end{tabular}

mosome $5 \mathrm{~B}$ or if three copies of the isochromosome for the long arm of chromosome $5 \mathrm{~B}$ are present. These differences in crossing over are discussed in section 4.5 .

6) Chromosome 5B has an effect on bouquet formation and its subsequent resolution. There is thus a more well defined and compact bouquet configuration in euploid wheat and diisosomic $5 \mathrm{BL}$ wheat than in nullisomic $5 \mathrm{~B}$ wheat. In the absence of chromosome 5B the bouquet also appears to resolve at an earlier stage of synapsis than in euploid wheat while in diisosomic and triisosomic 5BL wheat the bouquet configuration persists into diplotene.

\subsection{Trihaploid wheat and wheat-rye hybrids}

A summary of the data obtained from the analysis of trihaploid wheat and the wheat-rye hybrids is given in Tables II and III. The following conclusion can be drawn as to the effect of chromosome 5B and its long arm.

1) In trihaploid wheat obtained by cultivation of anthers from Chinese Spring wheat synaptonemal complex formation stops when about 35$40 \%$ of the complement has synapsed. Haploids derived from Kedong achieve up to $90 \%$ synapsis. As only two nuclei were analyzed from trihaploid wheat nullisomic for chromosome $5 \mathrm{~B}$ the extent of synapsis in this genotype cannot be

Table III. Degree of pairing, number of lateral components (LC) in multiple associations involving more than four LC's, number of pairing partner exchanges per LC engaged in synapsis and number of chiasmata per metaphase In wheat-rye hybrids without chromosome $5 B(0 \times 5 B)$, with $5 B(1 \times 5 B)$ or carrying an isochromosome for the long arm of chromosome 5B (2×5BL). EZ, early zygotene; MZ, mid zygotene; LZ, late zygotene; ED early diplotene; ND, not determined.

\begin{tabular}{|c|c|c|c|c|c|c|}
\hline \multicolumn{2}{|c|}{ Genotype } & \multirow{2}{*}{$\begin{array}{l}\begin{array}{l}\text { Number } \\
\text { of } \\
\text { nuclei }\end{array} \\
2\end{array}$} & \multirow{2}{*}{$\begin{array}{l}\text { pairing } \\
8\end{array}$} & \multirow{2}{*}{$\begin{array}{l}\begin{array}{l}\text { Number of LC's } \\
\text { in multiple } \\
\text { associations }\end{array} \\
25\end{array}$} & \multirow{2}{*}{$\begin{array}{l}\text { Number of pairing } \\
\text { partner exchanges } \\
\text { per LC }\end{array}$} & \multirow[t]{2}{*}{$\begin{array}{l}\text { Number of } \\
\text { chiasmata }\end{array}$} \\
\hline $0 \times 5 B$ & $\mathrm{EZ}$ & & & & & \\
\hline & $M Z$ & 8 & 15 & 29 & 1.14 & 6.9 \\
\hline & $\mathrm{LZ}$ & 12 & 34 & 28 & 1.07 & \\
\hline & $\mathrm{ED}$ & 2 & 52 & ND & ND & \\
\hline \multirow[t]{4}{*}{$1 \times 5 B$} & $\mathrm{EZ}$ & 3 & 13 & 29 & 1.14 & \\
\hline & $\mathbf{M Z}$ & 2 & 31 & 25 & 0.81 & 0.7 \\
\hline & $\mathrm{LZ}$ & 2 & 43 & 14 & 0.47 & \\
\hline & ED & 9 & 32 & ND & ND & \\
\hline \multicolumn{2}{|c|}{$2 \times 5 \mathrm{BL} \mathrm{EZ}$} & ND & ND & ND & ND & \\
\hline & $\mathbf{M Z}$ & 6 & 29 & 22 & 1.19 & $0.05 \S$ \\
\hline & $\mathrm{LZ}$ & 6 & 32 & 17 & 0.63 & \\
\hline & ED & 4 & 56 & ND & ND & \\
\hline
\end{tabular}

$\S$, Excluding the chiasmata in the isochromosome 
assessed. In each of the three wheat-rye hybrids analyzed there is substantial variation in the degree of synapsis between nuclei at the same stage. In all three genotypes some $40-50 \%$ of the complement synapses. Only the isochromosome achieves full synapsis.

2) In the absence of chromosome 5B in haploid wheat there are nearly 2.5 times more lateral components in associations involving more than three lateral components than in the euploid haploids. Likewise, there are nearly twice as many pairing partner exchanges per lateral component involved in synapsis in the absence of $5 \mathrm{~B}$ than in its presence. In the nullisomic 5B wheat-rye hybrids there are at late zygotene about twice as many lateral components in associations involving more than four lateral components than in the euploid hybrids and the hybrids carrying the isochromosome. The number of pairing partner exhanges per lateral component in nullisomic $5 \mathrm{~B}$ hybrids exceeds that found in the euploid hybrids by a factor of 2.3 and that in the monoisosomic 5BL hybrids by a factor of 1.7 .

3) In euhaploid wheat pairing partner exchanges are eliminated and several multiple associations corrected to associations of two and three chromosomes as pairing proceeds. This is also the case in the wheat-rye hybrids where the number of pairing partner exchanges by late zygotene is reduced to half of that found at earlier stages of zygotene. Likewise, with the exception of the nullisomic 5B hybrids where there are as many lateral components in multiple associations at late zygotene as at early zygotene, there are in the other two genotypes only half the number of lateral components at late zygotene than at the preceeding stages.

4)In spite of $35-40 \%$ synapsis in the trihaploid wheat derived from Chinese Spring; up to $90 \%$ synapsis in trihaploids obtained by cultivation of anthers from the variety Kedong and 40-50\% synapsis in the euploid and monoisosomic 5BL hybrids there is virtually no chiasma formation in these materials. In the absence of chromosome $5 \mathrm{~B}$ the number of chiasmata increases more than 40 times in the trihaploid wheat while there is an approximate ratio of 100:10:1 in the number of chiasmata in wheat-rye hybrids without $5 \mathrm{~B}$, with a single $5 \mathrm{~B}$ and with an isochromo- some for the long arm of chromosome 5B.

5)In the nullisomic 5B hybrids the bouquet appears to be more open and resolves at an earlier stage than in the euploid and monoisosomic 5BL hybrids.

\section{DISCUSSION}

\subsection{General aspects of synapsis in wheat}

The cultivar Chinese Spring is the standard variety for wheat cytogenetic studies. According to SEARS and Miller (69) this cultivar has not been selected for any particular reason save that it hybridizes readily with rye and major differences in the meiotic characteristics between this cultivar and other wheat cultivars are not apparent. The present analysis of the two Danish winter wheat varieties Vuka and Kraka, showing interlocking and pairing partner exchange at zygotene, incomplete synapsis leaving some interlockings and multiple associations unresolved and uncorrected and a short duration of the pachytene stage provides an initial indication that Chinese Spring also at the ultrastructural level may be considered representative for other wheat cultivars.

In contrast, major differences are apparent in the extent of synapsis in haploids derived from Chinese Spring and the Chinese winter wheat variety Kedong, where up to $90 \%$ synapsis was observed in the latter but only up to $41 \%$ in the former. It remains unknown whether differences exist also at the hexaploid level as synapsis in the hexaploid Kedong wheat has not been analyzed, but the difference at the trihaploid level may be attributed to the fact that the haploids from Kedong were much larger and vigorous than the haploids derived from Chinese Spring.

The incomplete synapsis in euallohexaploid wheat may, in addition to leaving a few multiple associations uncorrected (see section 4.5) and interlockings unresolved (20) have some further effect on meiosis. In the incompletely paired pachytene nuclei the unpaired segments are often of considerable length, comparable to the length of a chromosome arm. Extensive asynapsis of a pair of homologues may result in the lack of crossing over, the result being precocious separation where the univalents may undergo nondisjunction at anaphase $\mathrm{I}$. This may explain 
why univalents are regularly found at metaphase I and why monosomic lines are easily obtained in wheat. Incomplete synapsis may also affect the distribution of crossing over in the individual bivalents. As the middle segments of the bivalents are the ones most frequently unsynapsed, crossing over may occur less frequently in these segments. Recent investigations using structural, biochemical and genetic markers have thus indicated that crossing over primarily occurs distally in the chromosome arms of wheat. In the long arm of chromosome $6 \mathrm{~B}$ there is virtually no crossing over between the centromere and the nucleolus organizing region located about two-thirds of the length of the short arm away from the centromere (9). In the long arm of chromosome $5 \mathrm{~B}$ the proximal $36 \%$ of the arm has a genetic length of only $6 \mathrm{cM}$, the middle $25 \%$ of the arm a length of $25 \mathrm{cM}$, while the distal $38 \%$ was $53 \mathrm{cM}$ long (29). The incomplete proximal synapsis may relate to the timing of meiosis, in particular the short duration of the pachytene stage, but may also depend on the genetic constitution of the proximal segments, not supporting synapsis and subsequent crossing over. In particular the chromosomes of the B genome contain large blocks of proximal heterochromatin consisting of repeated DNA sequences (27) and synaptonemal complex formation appears often to be delayed or incomplete in large heterochromatic regions containing highly repeated DNA (54). Synapsis can also be incomplete in centromere regions devoid of centromeric heterochromatin as exemplified in Allium ursinum (42).

\subsection{Pairing arrest}

The incomplete synapsis in hexaploid wheat in which chromosome $5 \mathrm{~B}$ has been replaced by one, two or three copies of an isochromosome for the long arm have to be attributed to the absence of the short arm of chromosome 5B. This can also account for incomplete synapsis in plants lacking chromosome 5B altogether. In agreement with this several light microscopic studies have suggested the presence of a "pairing promoter" on this chromosome arm as wheat plants with one or two isochromosomes for $5 \mathrm{BL}$ have a somewhat lower number of chiasmata at metaphase I than euploid wheat or wheat tetrasomic for 5B $(58,62)$. This "pairing promoter" appears to reside in the distal third of the short arm of chromosome 5B as homozygosity for a deletion of this segment results in chiasma frequencies similar to that found in wheat lacking the entire short arms of chromosomes 5B (39). However, since the degree of pairing in nullisomic 5B wheat (35-40\%) and di- and triisosomic $5 \mathrm{BL}$ wheat ( $40 \%$ and $25 \%$ ) is somewhat lower than in monoisosomic 5BL wheat (55\%), the absence of the long arm or its presence in four or six copies may additionally reduce the degree of synapsis.

The relationship between dosage of the long arm of chromosome 5B and the degree of pairing achieved in the different hexaploid genotypes investigated is shown in Figure 1. On the

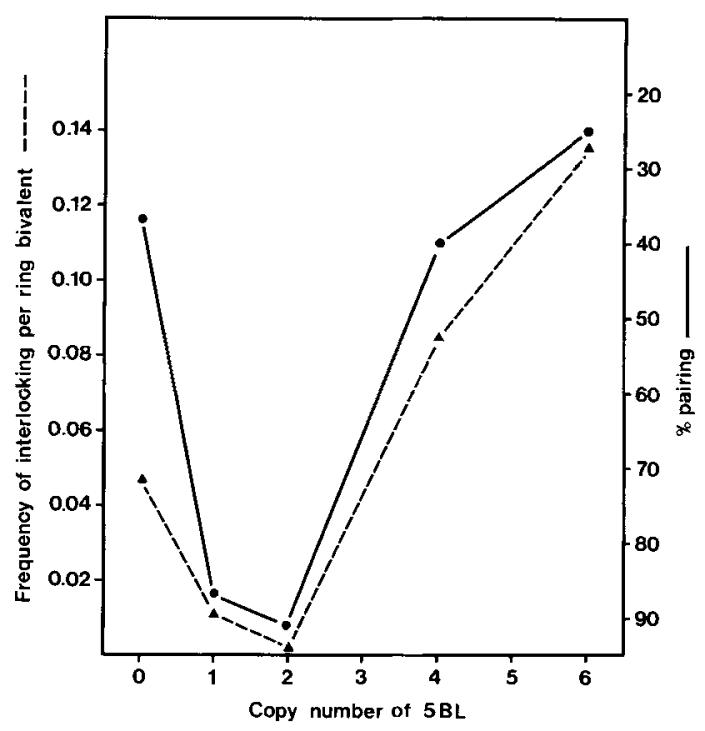

Figure 1. A graphical presentation of the relationship between the number of copies of 5BL and the degree of synapsis achieved by the different genotypes investigated in hexaploid wheat (continuous line) using the mean of the pairing percentage at pachytene and early diplotene. For triisosomic 5BL wheat the pairing percentage is from a single reconstructed nucleus. The data of YACOBI et al. (85) on the relationship between dosage of $5 \mathrm{BL}$ and interlocking frequency at metaphase I (expressed as frequency of interlocking per ring bivalent) is included for comparison (broken line). 
assumption that the absence of the short arm of chromosome $5 \mathrm{~B}$ reduces synapsis to the same extent in wheat diisosomic and triisosomic for $5 \mathrm{BL}$ there is an almost linear correlation, i.e. a possible dosage effect of the long arm on the degree of synapsis in the range from two to four to six copies of the long arm.

The other curve in Figure 1 (broken line) presents the data by YACOBI et al. (85) on the relationship between dosage of chromosome $5 \mathrm{BL}$ and the frequency of interlocking at metaphase I, expressed as the frequency of interlocking per ring bivalents. It is apparent (Figure 1) that there is an excellent correlation between the interlocking frequency and the amount of synapsis achieved in the five genotypes investigated. YACOBI et al. attributed the different frequencies of interlocking at metaphase I to a dosage effect of the $\mathrm{Ph}$ locus on a premeiotic organization of the chromosomes and genomes of wheat $(13,85)$. In the absence of $\mathrm{Ph}$ all homoeologues were assumed to be associated, the result being multivalent formation and interlocking between homoeologues. Two copies of $\mathrm{Ph}$ should result in a close association of homologues only whereby interlocking could not occur. Six doses of $\mathrm{Ph}$ should suppress all premeiotic associations, leading to a random distribution of chromosomes in the nucleus and result in interlocking and some synapsis between homoeologues. In contrast, the present data show that the interlocking frequency at metaphase I instead relates to the extent of synapsis achieved at zygotene in the individual genotypes. In organisms where the synapsis is complete all interlockings are usually resolved by the breakage-reunion mechanism (82). This mechanism appears to be activated when the lateral components in a region of interlocking are brought into contact either due to progression of synaptonemal complex formation between the interlocking lateral components or due to movement in different directions of the interlocked and interlocking chromosomes. When synapsis is arrested before completion several interlockings are not recognized and resolved and are maintained up to metaphase I. The present data reveal that for the investigated genotypes with partial asynapsis, the frequency of interlocking at metaphase I may be taken as a quantitative measure of the degree of synapsis achieved at zygotene.

In the three wheat-rye hybrid materials a similar effect on the extent of synapsis by the presence/absence of chromosome $5 \mathrm{~B}$ or the presence of an isochromosome is not apparent as all three hybrids appear to proceed roughly to the same amount of synapsis. The hexaploid and the hybrid materials may, however, not be directly comparable since synapsis in the hybrids only can occur between homoeologous and nonhomologous chromosomes. As nullisomy for chromosome $5 \mathrm{~B}$ results in an increased frequency of synapsis between homoeologous and nonhomologous chromosomes (see section 4.3), a higher degree of synapsis would be expected in the nullisomic 5B hybrids. The absence of the short arm of chromosome 5B may, however, counteract this effect.

Pairing arrest at early-mid zygotene is not per se a determining factor for the changes in the frequency of pairing partner exchanges in the different genotypes. In nullisomic 5B hexaploid wheat pairing is arrested when approximately the same degree of pairing is achieved as in wheat carrying two isochromosomes for the long arm of chromosome 5B, but there are four times more lateral components in multiple associations in the former genotype than in the latter. Likewise, the twofold difference in frequency of pairing partner exchanges per lateral component noted in the different materials of the wheat-rye hybrids is not correlated with similar differences in the degree of synapsis achieved.

\subsection{Specificity of synapsis}

Nullisomy for chromosome 5B results in hexaploid wheat, trihaploid wheat and wheatrye hybrids in a two- to threefold increase in the number of lateral components in multiple associations and the number of pairing partner exchanges per lateral component engaged in synapsis. These differences are apparent already from early zygotene. In hexaploid wheat there is an almost linear decrease in the number of lateral components in multiple associations when the number of copies of $5 \mathrm{~B}$ increases from 0 to 1 to 2 copies (Table I, Figure 2). The replacement of chromosomes $5 \mathrm{~B}$ with two 


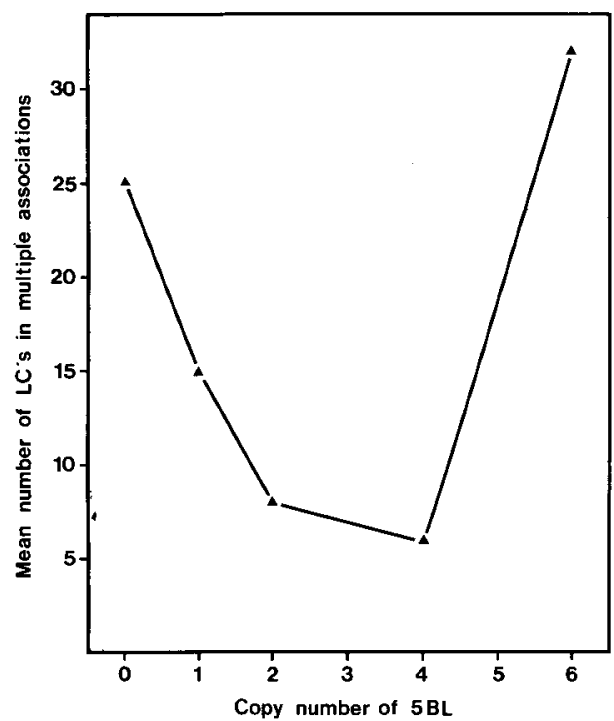

Figure 2. A graphical presentation of the relationship between copy number of $5 \mathrm{BL}$ and the number of lateral components in multiple associations at earlymid zygotene in the five investigated genotypes of hexaploid wheat.

isochromosomes for the long arm of $5 \mathrm{~B}$ results in a number of lateral components in multiple associations which is similar to or slightly lower than that seen in euploid wheat at the same stage of synapsis (Table I, Figure 2). However, while the results from the nullisomic, monosomic and euploid genotypes provide a measure of the amount of synapsis between chromosomes that are not homologous, the frequency observed in the diisosomic 5BL genotype includes possible pairing partner exchanges between the four identical/homologous arms of the two isochromosomes as these cannot be distinguished from the other lateral components of the complement. The actual frequency of synapsis between homoeologous and nonhomologous chromosomes may therefore be somewhat lower in this genotype than in euploid wheat. This may also be the case for the triisosomic 5BL genotype, where the presence of six homologous/identical arms may contribute significantly to the high frequency of pairing partner exchanges observed in this genotype.

A substantial part of the pairing partner exchanges in monosomic $5 \mathrm{~B}$ wheat may be at- tributed to the mere presence of a univalent chromosome $5 \mathrm{~B}$, resulting in pairing partner competition between the partly homologous chromosomes of homoeologue group 5 (21) rather than a dosage effect of chromosome 5B. A dosage effect of chromosome $5 \mathrm{~B}$ on the frequency of pairing partner exchange is not found in the three wheat-rye hybrids (Table III). As pairing partner exchanges also are very frequent in hexaploid wheat carrying three isochromosomes for the long arm of chromosome 5B, the breakdown of homologous synapsis in nullisomic $5 \mathrm{~B}$ and triisosomic $5 \mathrm{BL}$ wheat more likely results from a disturbance of the synaptic process caused by an absence or an excess number of copies of the $\mathrm{Ph}$ gene rather than a dosage effect of $\mathrm{Ph}$.

In all three types of materials (hexaploid, trihaploid and wheat-rye hybrids) the increase in the number of pairing partner exchanges in the absence of chromosome 5B is apparent already from the beginning of zygotene and can thus not be attributed to differences in correction of the multiple associations. Instead, the absence of chromosome $5 \mathrm{~B}$ appears to result in a relaxation of the stringency of synapsis. This contention is further supported by the observation that the location of the pairing partner exchanges as well as the types of multiple associations are different in the nullisomic 5B and euploid wheat. In the latter the multiple associations at early zygotene primarily result from short "synaptic contacts" between extensively synapsed bivalents (Type II, "switch back" associations) while pairing partner exchanges flanked by long segments of synaptonemal complex as well as pairing partner exchanges close to the telomeres are frequent in the absence of 5B. As analyses of nuclei at the leptotene to zygotene transition in euploid wheat have failed to reveal alignment of pairs or groups of lateral components, a premeiotic alignment of homologues as a means of ensuring synapsis preferentially between homologues is excluded.

It is frequently assumed that the specific synapsis between homologous segments at zygotene results from a recognition at the DNA level, perhaps by heteroduplex formation between single stranded DNA from the aligned chromosome segments. In Lilium evidence has 
thus accumulated that recognition between homologues is mediated by heteroduplex formation between a class of unique sequences (the socalled zygotene DNA), which remains unreplicated until zygotene (73). A similar hypothesis proposes that meiotic synapsis is mediated by the invasion of a large number of single stranded DNA segments ("feelers") from one chromosome meeting another one (71). Only if several neighbouring heteroduplexes can be formed in this way the synaptic configuration will persist, whereas a lack of extended homology results in a resolution of the heteroduplexes and the synaptic configuration with possible gene conversion but no chiasma formation as a consequence.

From ultrastructural observations $(19,82)$ it is apparent that synapsis of chromosomes results from at least two consecutive events, namely alignment and synaptonemal complex formation. During alignment juxtapositioned chromosomes are oriented parallel with each other at a distance larger than the width of the synaptonemal complex. Thereafter, synaptonemal complex formation may occur, being initiated by rotation of the chromatin relative to the lateral component. Precursor material for the central region is organized along this lateral component and following exposure of the other lateral component and its binding to the free face of the organized central region, the synaptonemal complex has formed. It is further a general observation from a large number of diploid and polyploid organisms that synapsis during early zygotene is a highly specific event, combining only homologous segments, while at pachytene synaptonemal complexes may form as readily between nonhomologous segments. These changes correlate with changes in chromosome movements, which at the beginning of zygotene are extensive but cease at pachytene (63). A reasonable hypothesis accounting for these findings could be that synaptonemal complex formation is entirely unspecific (17), its assembly being triggered in any region of alignment which has been maintained for a sufficient length of time. As a stable alignment at early zygotene with its vigorous movements is only possible in the presence of a high degree of homology between the aligned segments, synapsis will be specific while a stable alignment can occur between regions of low homology provided the chromosomes do not move. This hypothesis also explains why nonhomologous synapsis is occasionally observed at zygotene in regions where the chromosome movements have been impeded by interlocking $(24,54)$.

Analyses of spread nuclei from monosomic 5B and euhexaploid wheat revealed at the leptotene to zygotene transition that the individual lateral component aligns with several other lateral components over short distances along its length, whereby virtual "networks" of multiply aligned lateral components form. These alignment events may reflect mutual tests for homology between chromosomes which are in the vicinity of each other. However, as the distance between the aligned segments generally is larger than the width of the central region, synaptonemal complexes have probably not formed in these regions. By means of such tests for homology, homologous chromosomes may be aligned over longer distances and synaptonemal complex formation initiated. This will occur primarily from the telomeres which are brought into proximity and confined in their movements to a two dimensional space by attachment to a limited region of the nuclear envelope.

In the absence of $5 \mathrm{~B}$ the recognition process is less stringent permitting more frequent alignment and synaptonemal complex formation between homoeologues. This may be the result of less vigorous chromosome movements and a reduced treshold for homology recognition.

\subsection{Correction of pairing}

In all hexaploid genotypes analyzed, in trihaploids and in the wheat-rye hybrids, the number of pairing partner exchanges is generally higher at early zygotene than at later stages. However, only in euploid wheat are the pairing partner exchanges almost completely eliminated. In this study as well as the analysis of synaptic correction in the achiasmatic autotetraploid females of the silkworm, where correction from quadrivalents to bivalents is complete (55), one gets the impression that the correction process as well as the directionality of the correction primarily is driven by the ongoing process of synapsis. During the progression of synapsis, pairing partner 
exchanges appear to be detected when synaptonemal complex formation has reached the exchange region from both sides and provided that crossing over has not occurred the central region of the synaptonemal complex is dissolved on the one side of the region of pairing partner exchange and reformed on the other side between the alternative combination of lateral components (52, 53 see 82 for review). As recognition and synaptonemal complex formation between any combination of the four homologues in the autotetraploid Bombyx must be assumed to be equally effective, it is considered likely that the directionality of correction will be determined by the length of the synaptonemal complex segments on either side of the region of pairing partner exchange. The region of pairing partner exchange may therefore move through the shorter synaptonemal complex segment until the telomeres are reached. In allopolyploids such as wheat the directionality of the correction may also be influenced by the differences in homology between the chromosomes in the multiple associations. Alignment during correction at zygotene may thus occur more readily between homologous than between homoeologous and nonhomologous chromosomes, facilitating synaptonemal complex formation between homologues. Multiple associations of type II are easily removed, as they merely represent short regions of synaptic contacts between otherwise homologously synapsed chromosome pairs while the single interstitial exchanges, flanked by long segments of synaptonemal complex requires extensive synaptic correction which generally have to extend to the telomeres to achieve completion.

In the trihaploids and the wheat-rye hybrids the correction may likewise favour synaptonemal complex formation between chromosomes that share the highest degree of homology, i.e. the homoeologues. The formation of several associations of two and three lateral components at the end of zygotene may thus indicate extensive synapsis between homoeologues.

\subsection{The effect of chromosome $5 B$ on synapsis and crossing over}

The increase in the number of lateral components in multiple associations observed in nullisomic 5B wheat compared to the situation in euploid wheat cannot per se account for chiasma formation between the homoeologues. Crossing over between homoeologues do not occur in euploid wheat in spite of the retention of some pairing partner exchanges remaining at the pachytene stage where crossing over is assumed to occur. In monosomic 5B wheat crossing over do not occur between homoeologues, irrespective of substantial pairing partner exchange at pachytene between chromosomes presumed to be homoeologues. In euhaploid wheat only one chiasma is found in every six cells although up to $90 \%$ of the complement can be paired with synaptonemal complexes, of which a large fraction must be between homoeologues, while in the absence of chromosome $5 \mathrm{~B}$ a mean of 6.7 chiasmata per cell is found (79). In euploid, nullisomic 5B and monoisosomic 5BL hybrids between wheat and rye there is a tenfold difference between the mean number of chiasmata per cell in nullisomic 5B and euploid hybrids (6.9 and 0.7 chiasmata per cell) and a hundredfold difference between nullisomic 5B and monoisosomic 5BL hybrids (6.9 and 0.05 chiasmata per cell) even though the three genotypes achieve roughly the same degree of pairing.

The long arm of chromosome 5B accordingly has a dual effect on meiosis. If two copies are present synapsis occurs with a high stringency and crossing over is confined to homologous chromosomes while its absence leads to decreased stringency of synapsis and crossing over is permitted between partly homologous chromosomes. Six copies of chromosome 5BL also result in a relaxation of the specificity of synapsis and permissiveness for homoeologous crossing over. The question thus emerges whether the $\mathrm{Ph}$ locus on the long arm of chromosome 5B codes for a function common to synapsis and crossing over.

In hybrids between $T$. aestivum (carrying either the wild type allele $\mathrm{Ph}$ or the mutant allele phlb) and the tetraploid T. kotschyi there is extensive chiasma formation between the homoeologues (up to pentavalent) in plants carrying the ph $1 \mathrm{~b}$ allele while very few chiasmata form in the presence of $\mathrm{Ph}$ (66). A detailed ultrastructural study of three spread microsporocyte nuclei, two carrying the phlb and 
one the $\mathrm{Ph}$ allele, where about $90 \%$ of the complement had synapsed, revealed a two- to threefold increase in the frequency of lateral components and synaptonemal complexes in multiple associations in the two nuclei containing the ph $1 \mathrm{~b}$ allele compared to the nucleus with the wild type allele (16). Furthermore, synapsis appeared to proceed slower in plants with the $\mathrm{Ph}$ allele than in plants with ph $1 \mathrm{~b}$ as evidenced by a high proportion of spread nuclei with little synapsis in the former genotype. It is thus apparent that in the ph $1 \mathrm{~b}$ mutant, synapsis as well as crossing over occur more readily between chromosomes that are not homologous than in hybrids with the wild type allele, Ph. This indicates that both events indeed are affected by the $\mathrm{Ph}$ locus.

\subsection{Hypotheses on the regulation of synapsis and crossing over in allopolyploids \\ 4.6.1. Wheat}

Among the hypotheses to explain the mechanisms ensuring disomic inheritance in allopolyploid plants $(60,64,65,81)$ two proposals as to the effect of $\mathrm{Ph}$ on meiosis in wheat have recently been under debate, namely the hypothesis of FELDMAN and Avivi $(13,14,15)$ that $\mathrm{Ph}$ exerts its effect on a premeiotic arrangements of chromosomes and the hypothesis of HOBOLTH $(18,82)$ that $\mathrm{Ph}$ controls the timing of crossing over relative to the correction of the multiple associations. According to FELDMAN two copies of $\mathrm{Ph}$ would ensure a close premeiotic alignment of homologues and thereby account for the exclusive presence of bivalents and the absence of interlocking at metaphase I. This prediction is inçorrect as analyses of the leptotene-zygotene transition stage have failed to reveal such a large scale alignment $(20,21)$. Furthermore, formation of multiple associations as well as interlocking are regular features of the zygotene pairing in wheat, their absence at metaphase I resulting from a correction of the multiple associations into bivalents and the resolution of the interlockings (20). A second prediction from this theory, namely that the virtual absence of chiasmata in the trihaploids can be attributed to a lack of pairing between the homoeologues is also disproven, as there is extensive synaptonemal complex formation in the haploids, forming multiple associations as well as associations of two and three lateral components (79). The proposed effect of different dosage of $\mathrm{Ph}$ on premeiotic associations, a zero dose resulting in a close alignment of homologues and their homoeologues and six doses suppressing all premeiotic associations, is likewise not supported as the effects of different dosage of the long arm of chromosome 5B on chiasma formation and interlocking appear to result from pairing arrest at meiotic prophase (section 4.3).

Likewise, the hypothesis of HовоLтн $(18,82)$ suggesting that the $\mathrm{Ph}$ gene controls the timing of crossing over has also been disproven by the present series of investigations. According to this hypothesis the presence of two copies of $\mathrm{Ph}$ was suggested to ensure that crossing over first occurs after the correction is completed. It was expected that in the absence of $\mathrm{Ph}$ crossing over is permitted before the multivalents are corrected while the presence of six copies of $\mathrm{Ph}$ (in wheat carrying three copies of the long arm of chromosome 5B) should have postponed crossing over to a late stage, i.e. early diplotene, where the conditions for crossing over are suboptimal as degradation of the synaptonemal complex has started. Ultrastructural investigations of wheat triisosomic for 5BL have on the contrary shown that the reduction in chiasma frequency to half of that seen in euploid wheat correlates with pairing arrest at an early stage of zygotene (84). Secondly, there is as originally described by FELDMAN some chiasma formation between homoeologues in this genotype. Thirdly, in euploid wheat, and in particular in monosomic 5B wheat, multiple associations persist through the interval of crossing over, but no chiasmata form between the homoeologues. Finally, extrapolating from the theory, the absence of chiasmata in trihaploid wheat should be attributed to a correction of all paired configurations into univalents before crossing over occurred. This is not the case as no indication for a correction into univalents is found in trihaploid Chinese Spring wheat, in which up to $40 \%$ of the complement synapses. Instead, the multiple associations are maintained until degradation of the synaptonemal complex is initiated at the onset of diplotene. 
The present series of investigations have supported the proposal (20) that the disomic inheritance in allohexaploid wheat is ensured by: 1) A high stringency of chromosome pairing at zygotene, whereby primarily homologously paired bivalents primarily are formed. 2) A correction of chromosome pairing, whereby multiple associations are corrected into bivalents during zygotene and pachytene and 3) a suppression of crossing over between paired homoeologous segments. The $\mathrm{Ph}$ locus appears to affect the stringency;of synapsis as well as the suppression of crossing over between partly homologous segments, whereas synaptic correction appears to be driven by the ongoing process of synapsis.

\subsubsection{Lolium}

Correction of chromosome pairing combined with a control of the placement of crossovers in multivalents have also been considered to account for the disomic inheritance in hybrids between Lolium temulentum $(2 n=14)$ and Lolium perenne $(2 n=14)$, where the presence of "diploidising genes" in combination with the presence of accessory B chromosomes have an effect similar to that of the $\mathrm{Ph}$ gene in wheat. In the presence of four B chromosomes the allotetraploid hybrid behaves as a diploid showing bivalents, a few univalents, but no multivalents at metaphase I, while only univalents are seen at metaphase I in the diploid hybrid carrying two B chromosomes. In the absence of B chromosomes multivalents are present at metaphase $I$ in the allotetraploid and in the diploid the complement is at this stage almost exclusively present as bivalents $(11,12,40,74)$.

Serial sectioning and three dimensional reconstruction of zygotene and pachytene nuclei of diploid hybrids, an allotriploid and the allotetraploids with and without diploidising factors disclosed a correction of synapsis from multiple associations into bivalents during zygotene and pachytene $(31,32,33,34)$. However, in the diploid and the allotetraploids carrying the diploidising factors some multivalents persisted through the period of crossing over, while at metaphase I only univalents were present in the diploid and bivalents and univalents in the allotetraploid. It was concluded that B chromosomes and diploidising genes probably exerted their effect in Lolium hybrids by preventing crossing over between paired segments of homoeologues.

Synapsis between the chromosomes may, however, also be affected. Reconstructions of five pachytene nuclei from the diploid hybrid without the diploidising factors revealed the presence of seven almost completely synapsed bivalents where most of the length differences between the homoeologues, which differ in DNA content by about $50 \%$, had been accomodated by length adjustment of the lateral components (31). In contrast, analysis of the diploid hybrid with the diploidising factors showed that in the two late pachytene nuclei studied only $32 \%$ and $21 \%$ of the complement had synapsed (34). Reconstruction of a late zygotene nucleus of the allotetraploid hybrid without the diploidising factors revealed that 14 of the 28 chromosomes were in multiple associations while in the two reconstructed pachytene nuclei from the allotetraploid hybrid carrying the diploidising factors only a mean of 6.5 of the lateral components (excluding the $\mathrm{B}$ chromosomes, which formed a quadrivalent) were in multiple associations (33). Hence, in the absence of diploidising factors, synapsis occurs more readily between the homoeologues in the diploid and in the tetraploid the presence of more pairing partner exchanges indicates a similar less stringent pairing. Preliminary investigations of spread microsporocytes of the diploid hybrids with the diploidising factors have, however, disclosed that also in this genotype can synapsis be complete (JENKINS, personal communication).

\subsubsection{Effect of colchicine}

As described in sections 3.2 and 3.3 differences in the bouquet organization as well as the timing of the resolution of the bouquet are apparent between the different materials investigated. The bouquet is more open and resolves at an earlier stage in the absence of chromosome $5 \mathrm{~B}$ than in euploid materials while in diisosomic and triisosomic $5 \mathrm{BL}$ wheat the bouquet appears to persist into diplotene. These results indicate structural differences and/or differences in the 
timing of the intranuclear events such as chromosome movements depending on dosage of 5B.

As evidenced from time-lapse photomicrography of rat spermatocytes the application of colchicine during leptotene and zygotene stops chromosome movements (63). When applied during premeiotic interphase and early meiotic prophase, severe asynapsis results in organisms such as Lilium and T. aestivum $(2,7,8,76$, see 1 and 75 for a discussion of the colchicine sensitive stages). In $T$. aestivum injection of colchicine into tillers containing microsporocytes at premeiotic interphase or early meiotic prophase results in substantial asynapsis and a low frequency of chiasma formation between homoeologues. Driscoll et al. (8) thus reported mean numbers of 21.28 univalents, 10.32 bivalents and 0.02 quadrivalents at metaphase I. FELDMAN and AVIVI (15) found that if colchicine was applied at a concentration of $5 \times 10^{-4} \mathrm{M}$ or below, the chiasma frequency at metaphase I was reduced, several interlockings were present and in addition some chiasma formation between homoeologues was induced (an average of 0.16 chiasmata between homoeologues per cell), almost exclusively giving rise to heteromorphic bivalents. It was concluded that by the application of colchicine the action of six copies of the long arm of chromosome 5B could be phenocopied. In triisosomic 5BL wheat and nullisomic $5 \mathrm{~B}$ wheat, however, the number of chiasmata between homoeologues is several times higher than can be induced by colchicine $(0.77$ and 0.68 chiasmata between homoeologues per cell versus 0.16 chiasmata per cell in the colchicine treated material (15)).

As outlined in section 4.3 a reduction in chromosome movements at zygotene in hexaploid wheat probably results in a relaxation of the stringency of synapsis, which in allopolyploids like wheat may increase the number of pairing partner exchanges. The effect of colchicine may be merely to depolymerize cytoplasmic microtubules involved in movements of the attachment sites for the telomeres in the nuclear envelope, but may also be more complicated as biochemical studies of Lilium microsporocytes have demonstrated a colchicine binding protein, insensitive to vinblastine, in a heavy lipoprotein fraction, possibly residing in the nuclear envelope. In untreated cells this fraction is also associated with a DNA binding protein, the R-protein, which facilitates the renaturation of DNA and of possible significance to synapsis. Following colchicine treatment a drastic decrease in the binding of the R-protein to the heavy lipoprotein fraction was found, and probably also the total amount of the R-protein had decreased $(26,70)$.

It can at present not be excluded that the $\mathrm{Ph}$ locus affects chromosome movements and/or the synthesis of $\mathbf{R}$ protein. However, as colchicine treatment only appears to be able to induce a level of recombination between homoeologues, several orders of magnitude below that observed in nullisomic $5 \mathrm{~B}$, and wheat triisosomic for 5BL, such effects of $\mathrm{Ph}$ are considered less likely.

\subsection{The effect of $P h$}

The dual effect of $\mathrm{Ph}$ affecting the stringency of synapsis and homologous crossing over might be explained if the absence of $\mathrm{Ph}$ provides for a type of pairing which permits crossing over between homoeologues. A possible mechanism affected could be the process of length adjustment of paired lateral components. It is a common observation at late zygotene that synaptonemal complex formation between chromosomes of different length, resulting from differences in DNA content, as found in partly homologous chromosomes or duplication heterozygotes, involves an adjustment of the length of the two lateral components. In such heteromorphic bivalents a perfect equality in the length of the two lateral components is achieved irrespective of large initial differences $(31,35$, 46). This is also the case in wheat, since all chromosomes in multiple associations in most cases appear as perfectly synapsed as homologous chromosomes. As the length adjustment may result in a displacement of the homoeoalleles along the synaptonemal complex, crossing over may not be possible in such adjusted segments. There is, however, no indications in the present series of investigations for a difference in length adjustment in nullisomic $5 \mathrm{~B}$ and the other analyzed allohexaploid wheat 
genotypes.

Detailed suggestions as to the function of $\mathrm{Ph}$ await further analyses of the molecular aspects of synapsis and crossing over as well as a demonstration of that $\mathrm{Ph}$ is a single locus coding for a single protein or RNA species. A favourable experimental situation for exploring the molecular mechanisms of crossing over between chromosomes of partial homology exists, however, in yeasts. The lager brewing yeast, Saccharomyces carlsbergensis, appears to have a hybrid origin as it contains chromosomes which by genetic and molecular tests are homologous to those of $S$. cereviseae as well as chomosomes of partial homology to the S. cereviseae chromosomes $(48$, $50)$.

In a previous speculation (25) it was suggested that the absence of $\mathrm{Ph}$ results in the synthesis of a molecule which can trigger the recombination machinery in homoeologously synapsed segments. In the present context it is considered relevant to draw attention to the recent speculations on common steps in synapsis and non-reciprocal recombination during meiosis. It has thus been proposed that heteroduplex formation between homologous regions in otherwise nonhomologous chromosomes during synapsis in some cases can result in short gene conversions between linked, nonallelic repeated genes or unlinked repeated genes (51). In agreement with this proposal frequent gene conversion has been reported for heteroallelic genes inserted at different positions in the yeast genome $(36,41)$. These ideas have been extended by CARPENTER (4) who suggested that homologous synapsis is achieved by initial pairing and synaptonemal complex formation between juxtapositioned chromosomes followed by a check for extended sequence homology via a recombination nodule mediated gene conversion-like event. Provided extensive homology synaptonemal complex formation proceeds whereas low or no homology leads to a dissociation of the synaptonemal complex.

As the timewise separated processes of synapsis and crossing over are assumed both to be initiated by heterodupiex formation, this might provide the common link in the action of the $\mathrm{Ph}$ gene. Two copies of $\mathrm{Ph}$ wildtype gene are optimal for heteroduplex formation which insures a maximum of homologue pairing and limits crossing over to homologous segments. Lack of the product of the $\mathrm{Ph}$ gene or extra amounts from six copies of the gene gives rise to heteroduplexes between homoeologous segments, the outcome being a higher number of pairing partner exchanges and crossing over between segments of partial homology.

\section{ACKNOWLEDGEMENTS}

We are indebted to Professor DITER vON Wettstein, Dr. Søren W. Rasmussen and cand. scient. BENTE WISCHMANN for discussion and review of the manuscript. The expert technical assistance of BIBI STAMPE ANDERSEN, JEAN Sage, Nina Rasmussen and ANN-SOFI StEINHOLTZ is gratefully acknowledged. We would also like to thank Dr. CHRIs GiLliEs, Sydney and Dr. GLYN JENKINS, Aberystwyth for communicating results and ideas during the course of this study. The Royal Danish Academy of Sciences and Letters are thanked for having provided one of us (P.B.H.) with a Niels Bohr Scholarship. The work was also supported by grant BI6-E168-DK from the Commission of the European Communities.

\section{REFERENCES}

1. Bennett, M.D. J.B. Smith, S. Simpson \& B. Wells: Intranuclear fibrillar material in cereal pollen mother cells. Chromosoma 71, 289-332 (1979)

2. Bennett, M.D. L.A. Toledo \& H. Stern: The effect of colchicine on meiosis in Lilium speciosum cv. "Rosemede". Chromosoma 72, 175-189 (1979)

3. BREESE. E.L., E.J. LewIS \& G.M. Evans: Interspecies hybrids and polyploidy. Phil. Trans. R. Soc. Lond. B. $292,487-497$ (1981)

4. CARPENTER, A.T.C.: Gene conversion, recombination nodules and the initiation of meiotic synapsis. Bio Essays 6, 232-236 (1987)

5. DARlington, C.D.: Recent advances in cytology. J. \& A. Churchill Ltd. pp. 671 (1937)

6. Dover, G.A. \& R. RILEY: Prevention of pairing of homoeologous meiotic chromosomes of wheat by an activity of supernumerary chromosomes of Aegilops. Nature 240, 159-161 (1972)

7. Dover, G.A. \& R. RiLeY: The effect of spindle inhibitors applied before meiosis on meiotic chromosome pairing. J. Cell Sci. 12, 143-161 (1973) 
8. Driscoll, C.J., N.L. DARVEY \& H.N. Barber: Effect of colchicine on meiosis of hexaploid wheat. $\mathrm{Na}$ ture 216, 687-688 (1967)

9. DVOR̆ÁK, J \& K-C. CHEN: Distribution of nonstructural variation between wheat cultivars along chromosome arm 6Bp: Evidence from the linkage map and physical map of the arm. Genetics 106, 325333 (1984)

10. DvořÁ, J., K.-C. CHEN \& B. GIORGI: The C-band pattern of a Ph-mutant of durum wheat. Can. J. Genet. Cytol. 26, 360-363 (1984)

11. Evans.G.M.\& E.W. Davies: The genetics of meiotic chromosome pairing in Lolium temulentum $\times$ Lolium perenne tetraploids. Theor. Appl. Genet. $71,185-192$ (1985)

12. Evans, G.M. \& A.J. Macefield: Suppression of homoeologous pairing by $\mathbf{B}$ chromosomes in a Lolium species hybrid. Nature New Biol. 236, 110-111 (1972)

13. Feldman, M.: The effect of chromosomes 5B, 5D and $5 \mathrm{~A}$ on chromosomal pairing in Triticum aestivum. Proc. Nat. Acad. Sci. USA 55, $1447-$ I453 (1966)

14. FELDMAN, M.\& L. AvIVI: Ordered arrangement of chromosomes in wheat. In: Chromosomes Today, eds. M.D. Bennett, A. Gropp \& U. Wolf. 8, 181-189, Georg Allen \& Unwin (1984)

15. Feldman, $M$ \& L. Avivi: Genetic control of bivalent pairing in common wheat: the mode of $P h 1$ action. Kew Chromosome Conf. III (1988) in press

16. Gillies, C.B: The effect of $\mathrm{Ph}$ gene alleles on synaptonemal complex formation in Triticum aestivum $\times$ T. kotschyi hybrids. Theor. Appl. Genet. 74, 430-438 (1987)

17. Hasenkampf, C.A.: Synaptonemal complex formation in pollen mother cells of Tradescantia. Chromosoma 90, 275-284 (1984)

18. Hobolth, P.: Chromosome pairing in allohexaploid wheat var. Chinese Spring. Transformation of multivalents into bivalents, a mechanism for exclusively bivalent formation. Carlsberg Res. Commun. 46, 129-173 (1981)

19. Holm, P.B.: Ultrastructural characterization of meiosis. Biol. Skr. Dan. Vid. Selsk. 25, 39-90 (1985)

20. Holm, P.B.: Chromosome pairing and chiasma formation in allohexaploid wheat, Triticum aestivum analyzed by spreading of meiotic nuclei. Carlsberg Res. Commun. 51, 239-294 (1986)

21. HoLM, P.B.: Chromosome pairing and synaptonemal complex formation in hexaploid wheat, monosomic for chromosome 5B. Carlsberg Res. Commun. 53, 57-89 (1988)

22. HoLm, P.B.: Chromosome pairing and synaptonemal complex formation in hexaploid wheat, nullisomic for chromosome 5B. Carlsberg Res. Commun. 53, 91-110 (1988)

23. Holm, P.B.: Chromosome pairing and synaptonemal complex formation in hexaploid wheat, monoisosomic and diisosomic for the long arm of chromosome 5B. Carlsberg Res. Commun. 53, $111-133$ (1988)

24. Holm,P.B.\& S.W. RasmuSSEN: Chromosome pairing, recombination nodules and chiasma formation in diploid Bombyx spermatocytes. Carlsberg Res. Commun. 45, 483-548 (1980)

25. Holm, P.B., X. Wang \& B. WischmanN: An ultrastructural analysis of the effect of chromosome $5 B$ on chromosome pairing in allohexaploid wheat. Kew. Chromosome Conf. III, (1988) in press

26. HotTA, Y. \& J. SHEPARD: Biochemical aspects of colchicine action on meiotic cells. Molec. gen. Genet. 122, 243-260 (1973)

27. HuTCHINSON, J.\& D.M. LONSDALE: The chromosomal distribution of cloned highly repetitive sequences from hexaploid wheat. Heredity 48, 371376 (1982)

28. Hutchinson, J., T.E. Miller \& S.M. Reader: Cbanding at meiosis as a means of assessing chromosome affinities in the Triticeae. Can. J. Genet. Cytol. 25, 319-323 (1983)

29. Jampates, R. \& J. DVOŘÁ: Location of the $P h l$ locus in the metaphase chromosome map and the linkage map of the $5 \mathrm{~Bq}$ arm of wheat. Can. J. Genet. Cytol. 28, 511-519 (1986)

30. Jenkins, G.: Chromosome pairing in Triticum aestivum cv. Chinese Spring. Carlsberg Res. Commun. 48, 255-283 (1983)

31. Jenkins, G.: Synaptonemal complex formation in hybrids of Lolium temulentum $\times$ Lolium perenne (L.). I. High chiasma frequency diploid. Chromosoma 92, 81-88 (1985)

32. JenKins, G.: Synaptonemal complex formation in hybrids of Lolium temulentum $\times$ Lolium perenne (L.). II. Triploid. Chromosoma 92, 387-390 (1985)

33. JENKINS, G.: Synaptonemal complex formation in hybrids of Lolium temulentum $\times$ Lolium perenne (L.). III. Tetraploid. Chromosoma 93, 413-419 (1986)

34. Jenkins, G.: Chromosome pairing in a Lolium temulentum $\times$ Lolium perenne diploid hybrid with a low chiasma frequency. Theor. Appl. Genet. 73, 516-522 (1987)

35. Jenkins, G. \& H. ReEs: Synaptonemal complex formation in a Festuca hybrid. Kew Chromosome Conference II, 232-242 (1983)

36. Jinks-Robertson, S. \& T.E. PeTERS: High-frequency meiotic gene conversion between repeated genes on nonhomologous chromosomes in yeast. Proc. Natl. Acad. Sci. USA. 82, 3350-3354 (1985) 
37. KIHARA, H. \&. OnO: Chromosomenzahlen und systematische Gruppierung der Rumex-Arten. Z. Zellforsch. 4, 475-481(1926)

38. KOEBNER, R.M.D. \& K.W. SHEPHERD: Induction of recombination between rye chromosome IRL and wheat chromosomes. Theor. Appl. Genet. 71, 208-215 (1985)

39. KOTA, R.S. \& J. DVOŘAK: Mapping of a chromosome pairing gene and $5 S$ rRNA genes in Triticum aestivum $\mathrm{L}$. by a spontaneous deletion in chromosome arm 5Bp. Can. J. Genet. Cytol. 28, 266-271 (1986)

40. LewIS, E.J.: Chromosome pairing in tetraploid hybrids between Lolium perenne and L. multiflorum. Theor. Appl. Genet. 58, 137-143 (1980)

41. LiChten, M., R.H. Borts \& J.E. Haber: Meiotic gene conversion and crossing over between dispersed homologous sequences occurs frequently in Saccharomyces cerevisiae. Genetics 115, 233-246 (1987)

42. LoIDL, J.: Synaptonemal complex spreading in Allium ursinum: Pericentric asynapsis and axial thickenings. J. Cell Sci. 87, 439-448 (1987)

43. Mello-Sampayo, T:: Homoeologous chromosome pairing in pentaploid hybrids of wheat. Proc. $3 \mathrm{rd}$ International Wheat Genetics Symposium pp. 179-184 (1968)

44. Mello-Sampayo, $T$ : Genetic regulation of meiotic chromosome pairing by chromosome $3 \mathrm{D}$ of Triticum aestivum. Nature New Biology 230, 2223 (1971)

45. Miller, T.E., S.M. REadER \& M.D.GALE: The effect of homoeologous group 3 chromosomes on chromosome pairing and crossability in Triticum aestivum. Can. J. Genet. Cytol. 25, 634-641 (1983)

46. Moses, M.J.\& P.A. PoORMaN: Synaptonemal complex analysis of mouse chromosomal rearrangements II. Synaptic adjustment in a tandem duplication. Chromosoma 81, 519-535 (1981)

47. Navashin, M.S.: Uber die Veränderung von Zahl und Form der Chromosomen infolge der $\mathrm{Hy}$ bridization. Z. Zellforsch. 6, 195-233 (1927)

48. Nillson-Tillgren, T., M.C. Kielland-Brandt, S. Holmberg, J.G.L. Pedersen \& C. Giermansen: Is lager yeast a species hybrid? Proc. IV. Symp. Genet. Industr. Microorg. Kyoto pp. 143-147 (1982)

49. OKamoto, M.: Asynaptic effect on chromosome V. Wheat Inf. Serv, 5, 6-7 (1957)

50. Petersen, J.G.L., T. Nillsson-Tillgren, M.C. Kielland-Brandt, C. Gjermansen \& S. HolmBERG: Structural heterozygosis at genes ILV2 and ILVS in Saccharomyces carlsbergensis. Curr. Genet. 12, 167-174 (1987)

51. Powers, P.A. \& O. SMithies: Short gene conver- sions in the human fetal globin gene region: $A$ by-product of chromosome pairing during meiosis. Genetics 112, 343-358 (1986)

52. RASMUSSEN, S.W.: Chromosome pairing in triploid females of Bombyx mori analyzed by three-dimensional reconstructions of synaptonemal complexes. Carlsberg Res. Commun. 42, 163-197 (1977)

53. Rasmussen, S.W.: Chromosome pairing in autotetraploid bombyx males. Inhibition of multivalent correction by crossing over. Carlsberg Res. Commun. 52, 211-242 (1987)

54. RaSMUSSEN, S.W.\& P.B. Holm: Human meiosis II. Chromosome pairing and recombination nodules in human spermatocytes. Carlsberg Res. Commun. 43, 275-327 (1978)

55. Rasmussen, S.W.\& P.B. Holm: Chromosome pairing in autotetraploid Bombyx females. Mechanism for exclusive bivalent formation. Carlsberg Res. Commun. 44, 101-125 (1979)

56. Riley, R: The diploidisation of polyploid wheat. Heredity 15, 407-429 (1960)

57. Riley, R \& V. Chapman: Genetic control of the cytologically diploid behaviour of hexaploid wheat. Nature 182, 713-715 (1958)

58. RiLey, R. \& V.ChaPMAN: Effect of $5 \mathrm{~B}^{\mathrm{s}}$ in suppressing the expression of altered dosage of $5 \mathrm{~B}^{\mathrm{L}}$ on meiotic chromosome pairing in Triticum aestivum. Nature 216, 60-62 (1967)

59. Riley, R. \& C. Kempanna: The homoeologous nature of the non-homologous meiotic pairing in Triticum aestivum deficient for chromosome $\mathrm{V}$ (5B). Heredity 18, 287-306 (1963)

60. RILEY,R \& C.N.LAW: Genetic variation in chromosome pairing. Adv. Genet. 13, 57-114 (1965)

61. RILEY, R., V.CHAPMAN \& R. JOHNSON: The incorporation of alien disease resistance in wheat by genetic interference with the regulation of meiotic chromosome synapsis. Genet. Res. 12, 199-219 (1968)

62. Riley, R., V. Chapman, R.M. Young \& A.M. BELFIELD: Control of meiotic chromosome pairing by the chromosomes of homoeologous group 5 of Triticum aestivum. Nature 212, 1475-1477 (1966)

63. Salonen, K., J. Paranko \& M: Parvinen: A colcemid-sensitive mechanism involved in regulation of chromosome movements during meiotic pairing. Chromosoma 85, 611-618 (1982)

64. SEARS, E.R.: Wheat cytogenetics. Ann. Rev. Genet. 3, 451-468 (1969)

65. SEARS, E.R.: Genetic control of chromosome pairing in wheat. Ann. Rev. Genet. 10, 31-51 (1976)

66. SEARS, E.R: An induced mutant with homoeologous pairing in common wheat. Can. J. Genet. Cytol. 24, 715-719 (1982)

67. Sears, E.R.: A wheat mutation conditioning an 
intermediate level of homoeologous chromosome pairing. Can. J. Genet. Cytol. 24, $715-719$ (1982)

68. SEARS, E.R.: The transfer to wheat of interstitial segments of alien chromosomes. Proc. 6th International Wheat Genetics Symposium, 5-12 (1983)

69. SEARS, E.R.\& T.E. Miller: The history of Chinese spring wheat. Cereal Res. Commun. 13, 261-263 (1985)

70. Shepard, J., E.R. Boothroyd \& H. Stern: The effect of colchicine on synapsis and chiasma formation in microsporocytes of Lilium. Chromosoma 44, 423-437 (1974)

71. SMithies, O. \& P.A. Powers: Gene conversions and their relation to homologous chromosome pairing. Phil. Trans. R. Soc. Lond. B. 312, 291-302 (1986)

72. Stebrins, G.L.: Types of polyploids: Their classification and significance. Adv. Genet. 1, 403-427 (1947)

73. Stern. H. \& Y. HotTA: The biochemistry of meiosis. In: Meiosis. ed. P.B. Moens pp 303-329, Academic Press (1987)

74. TAYLOR, J.B. \& G.M. EVANS: The genotypic control of homoeologous chromosome association in Lolium temulentum $\times$ Lolium perenne. Interspecific hybrids. Chromosoma 62, 57-67 (1977)

75. Thomas, J.B. \& P.J. Kaltsikes: The effect of colchicine on chromosome pairing. Can. J. Genet. Cytol. 19, 231-249 (1977)

76. Toledo, L.A., M.D. BenNETt \& H. Stern: Cytological investigation of the effect of colchicine on meiosis in Lilium hybrid cv. 'Black Beauty' microsporocytes. Chromosoma 72, 157-173 (1979)
77. WALl. A.M., R. Riley \& V. ChapMaN: Wheat mutants permitting homoeologous meiotic chromosome pairing. Genet. Res. Camb. 18, 311-328 (1971)

78. WALL, A.M., R. RILEY \& M.D.GALE: The position of a locus on chromosome $5 \mathrm{~B}$ of Triticum aestivum affecting homoeologous meiotic pairing. Genet. Res. Camb. 18, 329-339 (1971)

79. WANG, X.: Chromosome pairing analysis in haploid wheat by spreading of meiotic nuclei. Carlsberg Res. Commun. 53, 135-166 (1988)

80. WANG, X.\& P.B. Holm: Chromosome pairing and synaptonemal complex formation in wheat-rye hybrids. Carlsberg Res. Commun. 53, 167-190 (1988)

81. Watanabe, K: Studies on the control of diploidlike meiosis in polyploid taxa of Chrysanthemum I. Hexaploid Ch. japonense Nakai. Cytologia 46, 459-498 (1981)

82. Wettstein, D. von, S.W. RasmusSEn \& P.B. Holm: The synaptonemal complex in genetic segregation. Ann. Rev. Genet. 18, 331-431 (1984)

83. WINGE, Ø.: The chromosomes, their numbers and general importance. C.R. Trav. Lab. Carlsberg 13, 131-275 (1917)

84. Wischmann, B.: Chromosome pairing and chiasma formation in wheat plants triisosomic for the long arm of chromosome 5B. Carlsberg Res. Commun. 51, 1-25 (1986)

85. Yacobi, Y.Z., T. Mello-Sampayo \& M. Feldman: Genetic induction of bivalent interlocking in common wheat. Chromosoma 87, 165-175 (1982) 